\title{
Pruebas presuntivas del análisis de orina en el diagnóstico de infección en vías urinarias entre diabéticos tipo 2
}

\author{
Eugenia Flores-Alfaro, MSP,(I) Isela Parra-Rojas, QBP,(I) \\ Angelino Jiménez-Acevedo, $Q B P^{(1)}$ Gloria Fernández-Tilapa, MC(1)
}

\begin{abstract}
Flores-Alfaro E, Parra-Rojas I, Jiménez-Acevedo A, Fernández-Tilapa G. Pruebas presuntivas del análisis de orina en el diagnóstico de infección en vías urinarias entre diabéticos tipo 2. Salud Publica Mex 2005;47:376-380.
\end{abstract}

\section{Resumen}

Objetivo. Determinar la confiabilidad de las pruebas presuntivas del análisis de orina en el diagnóstico temprano de infecciones de vías urinarias entre pacientes con diabetes tipo 2. Material y métodos. En el año 200I se realizó un estudio transversal entre 160 diabéticos con diagnóstico clínico de infección en vías urinarias, de la ciudad de Tlapa, Guerrero. Se confirmó este diagnóstico por urocultivo, se comparó con las pruebas presuntivas de esterasa leucocitaria, nitritos, cuenta de leucocitos y bacteriuria en orina centrifugada y sin centrifugar. Se calculó la sensibilidad, la especificidad, el valor predictivo positivo y el negativo. Resultados. En $31 \%$ de los participantes se confirmó infección urinaria. La presencia de bacterias en orina centrifugada, esterasa leucocitaria y la cuenta de leucocitos presentaron sensibilidad y especificidad altas. Conclusiones. Las mediciones de esterasa leucocitaria y bacteriuria en orina centrifugada ofrecen confiabilidad en el diagnóstico oportuno de infecciones de vías urinarias.

Palabras clave: técnicas y procedimientos de laboratorio; infecciones urinarias; diabetes mellitus; México
Flores-Alfaro E, Parra-Rojas I,

Jiménez-Acevedo A, Fernández-Tilapa G.

Presuntive tests in screening for detecting

urinary tract infections in patients with diabetes type-2.

Salud Publica Mex 2005;47:376-380.

\section{Abstract}

Objective. To determine the validity of the presumptive tests used to analyze urine in order to detect urinary tract infections in patients with diabetes mellitus type-2. Material and Methods. In 200I, a cross-sectional study was carried out in 160 patients with diabetes mellitus type-2 who were clinically diagnosed with urinary tract infections. Patients were from Tlapa, which is located in the state of Guerrero. Urine samples were microscopically analyzed to determine the number of leukocytes and bacteria in centrifuged samples and urine without centrifuge. The leukocyte esterasa and nitrites were measured using reactive strips. All samples were subjected to urine culture to confirm urinary tract infections. Sensitivity, specificity, and predictive values were calculated for leukocyte count, bacteriuria, leukocyte esterasa and nitrites using positive urine cultures as the standard of measurement. Results. Urinary tract infections were confirmed in $31 \%$ of patients. The presence of bacteria in centrifuged urine, leukocyte esterasa and the leukocyte count were all high with respect to sensitivity and specificity. Conclusions. Leukocyte esterasa and microscopic bacteriuria measurements in centrifuged urine offer reliability in the early diagnosis of urinary tract infections.

Key words: laboratory techniques and procedures; urinary tract infection; diabetes mellitus, Mexico

(I) Facultad de Ciencias Químico Biológicas, Universidad Autónoma de Guerrero. Chilpancingo, Guerrero, México.

Fecha de recibido: 25 de noviembre de 2004 - Fecha de aprobado: 17 de agosto de 2005 Solicitud de sobretiros: Eugenia Flores-Alfaro. Laboratorio de Enfermedades Crónico Degenerativas. Facultad de Ciencias Químico Biológicas. Avenida Lázaro Cárdenas s/n. 39090, Ciudad Universitaria. Chilpancingo, Guerrero, México. Correo electrónico: efloresa@todito.com 
L as infecciones de vías urinarias (IVU) producen cistitis, uretritis y prostatitis en vías bajas, y pielonefritis en vías altas; se encuentran relacionadas con problemas obstructivos, alteraciones en la función del tracto urinario (TU), enfermedades sistémicas, embarazo, menopausia, resistencia antimicrobiana, uso de catéteres, lesiones químicas o por radiación del TU, entre otros factores. ${ }^{1,2}$ Se registra un espectro amplio de agentes causales de IVU, pero Escherichia coli es la más frecuente. ${ }^{3}$

La diabetes mellitus (DM) es un factor predisponente para adquirir infección en vías urinarias. ${ }^{4-8}$ En México, la Encuesta Nacional de Salud reporta una prevalencia de DM de $7.5 \%$ en los individuos mayores de 20 años de edad, ${ }^{9}$ y la Secretaría de Salud la reconoce como la primera causa de muerte. ${ }^{10}$ Los pacientes diabéticos tienen dos veces más riesgo de adquirir infecciones complicadas del tracto urinario en comparación con los que no la padecen; la pielonefritis aguda es cinco veces más frecuente en diabéticos; en $60 \%$ de los pacientes hospitalizados con bacteremia y diabetes, la fuente de infección son las vías urinarias. ${ }^{7}$ En el estudio de casos y controles realizado por Goswami y colaboradores se notifica una prevalencia de $9 \%$ de IVU en diabéticos. ${ }^{11}$ El 90\% de los casos de abscesos perinefríticos y pielonefritis enfisematosa ocurren en diabéticos mal controlados; las infecciones urinarias son frecuentes en diabéticas embarazadas y generalmente se presentan asintomáticas. ${ }^{4}$ Se ha notificado que las mujeres diabéticas bajo tratamiento médico con hipoglicemiantes o insulina tienen 2.9 y 2.6 veces más riesgo, respectivamente, de presentar infecciones urinarias en comparación con mujeres no diabéticas. ${ }^{5}$ Las IVU se relacionan con la descompensación y elevación de la glicemia, pueden evolucionar hacia la cronicidad y, por tanto, promover fallo renal y consecuentemente un deterioro de la calidad de vida de los pacientes que la padecen. ${ }^{12,13}$

La mayoría de las IVU son producidas por microorganismos Gram negativos; la mayor frecuencia corresponde a los serotipos de $E$. coli, ${ }^{3,14}$ responsables de más de $80 \%$ de las infecciones primarias y de $75 \%$ de las recurrencias. ${ }^{5}$ El análisis químico y microscópico de la orina, además de sencillo y económico, es rápido y útil como alternativa de diagnóstico presuntivo oportuno. El uso de las tiras reactivas en el diagnóstico de infecciones urinarias es un método útil para el médico: puede usarlo en la práctica diaria y obtener un diagnóstico previo que confirmará posteriormente el laboratorio. ${ }^{15}$

Considerando la importancia que reviste el diagnóstico oportuno de infecciones urinarias en pacientes con diabetes mellitus tipo 2, en esta investigación se evaluó la confiabilidad de: la prueba de bacteriuria en orina centrifugada y sin centrifugar utilizando tinción de Gram; la determinación de nitritos y de esterasa leucocitaria por tira reactiva, y la cuenta de leucocitos en cámara de Neubauer, con el fin de identificar qué pruebas pueden ser más confiables para el diagnóstico temprano de IVU en pacientes diabéticos.

\section{Material y métodos}

En el año 2001 se realizó un estudio transversal entre 160 pacientes diabéticos tipo 2, captados en el Laboratorio Bioquímico de la ciudad de Tlapa de Comonfort, Guerrero. Se incluyeron personas mayores de 30 años, de ambos sexos, con diagnóstico probable de IVU y/o presencia de alguno de los síntomas característicos: dolor al orinar, dolor en región costolumbar y micción frecuente. Se excluyeron los pacientes que se encontraban con tratamiento antimicrobiano por éste $\mathrm{u}$ otro proceso infeccioso. Las personas que aceptaron participar en el estudio, lo hicieron mediante consentimiento informado. El proyecto fue aprobado por el Comité de Bioética de la Dirección de Investigación Científica de la Universidad Autónoma de Guerrero

A cada participante se le solicitó una muestra de orina del "chorro medio" de la micción en frasco estéril, previa asepsia de los genitales externos con agua estéril o hervida; las muestras fueron entregadas al Laboratorio Bioquímico para su procesamiento.

El urocultivo fue la prueba de oro para determinar IVU. Las muestras de orina se sembraron en agar soya tripticasa, gelosa sangre, sal y manitol, Biggy y EMB. El medio agar soya tripticasa se sembró con asa calibrada 1:1000 (0.001 ml) extendiendo la muestra en forma de rejilla para el recuento de colonias de microorganismos; en el resto de los medios se colocaron 25 $\mu l$ de orina homogenizada y se extendieron por estría cruzada. Todos los medios se incubaron a $37^{\circ} \mathrm{C}$ por 24 horas, al cabo de las cuales se verificó el crecimiento y se hizo el conteo de colonias. Se determinó IVU cuando el cultivo presentó $\geq 100,000$ UFC / ml. Los microorganismos se identificaron por pruebas bioquímicas convencionales. Se probó in vitro la sensibilidad a los antibióticos en agar de Muller-Hinton aplicando sensidiscos.

El recuento de leucocitos se determinó por cámara de Neubauer utilizando $12 \mathrm{ml}$ de orina; la prueba se consideró positiva cuando la cuenta fue de cinco o más leucocitos por $\mathrm{mm}^{3}$. En las muestras de orina sin centrifugar se determinaron nitritos y esterasa leucocitaria por medio de tiras reactivas Multistix. Se preparó un frotis con $25 \mu \mathrm{l}$ de orina homogeneizada y otro de sedimento urinario; ambos se tiñeron con tinción de 
Gram y se observaron al microscopio. Se tomó como prueba positiva la presencia de al menos una bacteria por campo de inmersión en 20 campos observados.

Los resultados se entregaron en forma escrita a los pacientes, y aquellos que presentaron IVU se remitieron a revisión y tratamiento médico.

Se obtuvieron las frecuencias simples de IVU y de los agentes microbianos que se identificaron; la confiabilidad de las pruebas presuntivas del análisis de orina se determinó por el cálculo de sensibilidad, especificidad, valor predictivo positivo (VPP) y negativo (VPN) e intervalos de confianza.

\section{Resultados}

El promedio de edad de las personas estudiadas fue de 50 años; $42.5 \%$ eran hombres, y $57.5 \%$, mujeres; $31 \%$ presentó IVU, y los microorganismos encontrados con mayor frecuencia fueron Staphylococcus aureus y E. coli (figura 1).

En el cuadro I se indican las frecuencias absolutas de las pruebas del análisis de orina en presencia de urocultivo positivo o negativo; en el cuadro II se muestran las determinaciones de sensibilidad, especificidad, VPP, VPN e intervalos de confianza de las pruebas presuntivas del análisis de orina. La prueba de bacteriuria con orina centrifugada presentó una sensibilidad de $100 \%$, seguida de la esterasa leucocitaria y de la cuenta de leucocitos, ambas con $90 \%$ de sensibilidad. Los nitritos presentaron la más alta especificidad $(100 \%)$.

Se evaluó la confiabilidad de la ocurrencia de tres o más pruebas del uroanálisis positivas en los pacien-

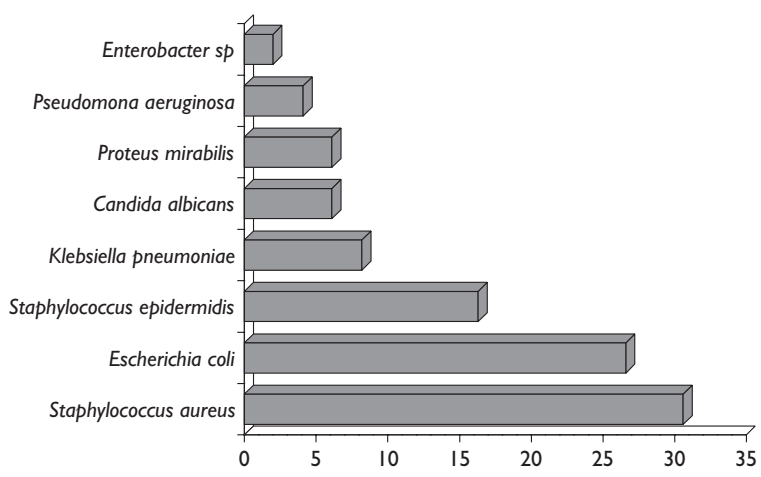

Figura I. Frecuencia de microorganismos causanTES DE IVU EN PACIENTES DIABÉTICOS TIPO 2. TLAPA DE Comonfort, Guerrero, México 2001
Cuadro I

Frecuencias absolutas de las pruebas presuntivas DEL ANÁLISIS DE ORINA EN RELACIÓN CON EL UROCULTIVO. Pacientes diabéticos tipo 2 de Tlapa de Comonfort, Guerrero. México, 200 I

Prueba presuntiva $\frac{\text { Urocultivo }}{\text { Positivo Negativo }}$

Bacteriuria (orina centrifugada)

\begin{tabular}{lrl} 
Positiva & 51 & 20 \\
\hline Negativa & 0 & 89
\end{tabular}

Esterasa leucocitaria (tira reactiva)

\begin{tabular}{lrr} 
Positivo & 45 & 4 \\
\hline Negativo & 5 & 106 \\
$\begin{array}{l}\text { Cuenta de leucocitos (cámara de Neubauer) } \\
\text { Positivo }\end{array}$ & 45 & 7 \\
\hline Negativo & 5 & 103
\end{tabular}

Nitritos (tira reactiva)

\begin{tabular}{lrr} 
Positivo & 18 & 0 \\
\hline Negativo & 32 & 110
\end{tabular}

Bacteriuria (orina sin centrifugar)

\begin{tabular}{lrr} 
Positiva & 45 & 25 \\
\hline Negativa & 6 & 84 \\
\hline
\end{tabular}

tes que las presentaron, y se encontró una sensibilidad de $84.3 \%$, especificidad de $100 \%$, VPP de $100 \%$ y VPN de $93.2 \%$.

\section{Discusión}

No se tiene información sobre la utilidad clínica de las diferentes pruebas del análisis de orina en el diagnóstico presuntivo de IVU entre población diabética; estos estudios son notificados en poblaciones diversas, principalmente entre mujeres embarazadas. ${ }^{16,17}$ Así, para la prueba con tira reactiva global, Lumbiganon y colaboradores registran una sensibilidad de $13.9 \%$, una especificidad de $95.6 \%$, un VPP de $46.1 \%$ y un VPN de $80.6 \%$ en mujeres embarazadas con bacteriuria asintomática, y concluyen que esta prueba tiene poca utilidad; ${ }^{16}$ estos resultados son similares a los notificados por Tincello y Richmond. ${ }^{17}$ Por el contrario, Uncu y colaboradores encontraron que la sensibilidad y la especificidad para leucocituria fueron de $91.3 \%$ y $83.6 \%$, respectivamente; concluyeron que esta prueba es de utilidad para el 


\section{Cuadro II \\ VALIDEZ Y CONFIABILIDAD DE LAS PRUEBAS PRESUNTIVAS DEL ANÁLISIS DE ORINA UTILIZADAS EN EL DIAGNÓSTICO DE INFECCIÓN DE VÍAS URINARIAS, ENTRE PACIENTES DIABÉTICOS TIPO 2. Tlapa de Comonfort, Guerrero. México, 2001}

\begin{tabular}{|c|c|c|c|c|}
\hline Prueba presuntiva & $\begin{array}{l}\text { Sensibilidad } \\
\% \text { (IC 95\%) }\end{array}$ & $\begin{array}{l}\text { Especificidad } \\
\% \text { (IC 95\%) }\end{array}$ & $\begin{array}{c}\text { VPP } \\
\% \text { (IC 95\%) }\end{array}$ & $\begin{array}{c}\text { VPN } \\
\% \text { (IC 95\%) }\end{array}$ \\
\hline Bacteriuria (orina centrifugada) & 100 & $81.7(75.6-87.6)$ & $71.8(64.8-78.8)$ & 100 \\
\hline Esterasa leucocitaria (por tira reactiva) & $90(85.4-94.7)$ & $96.4(93.4-99.2)$ & $91.8(87.5-96.1)$ & $95.5(92.2-98.6)$ \\
\hline Cuenta de leucocitos (cámara de Neubauer) & $90(85.4-94.7)$ & $93.6(89.8-97.4)$ & $86.5(81.2-91.8)$ & $95.4(92.0-98.6)$ \\
\hline Nitritos (por tira reactiva) & $36(28.6-43.4)$ & 100 & 100 & $77.5(70.9-83.9)$ \\
\hline Bacteriuria (orina sin centrifugar) & $88.2(83.2-93.2)$ & $77.1(70.5-83.5)$ & $64.3(56.8-71.6)$ & $93.3(89.3-97.2)$ \\
\hline
\end{tabular}

diagnóstico de IVU en estas mujeres, ${ }^{18}$ lo que coincide con los estudios de otros investigadores. ${ }^{20-22}$ En varias investigaciones se observa que, además de determinar la esterasa leucocitaria por tira reactiva se efectúa la medición de leucocitos en cámara de Neubauer para incrementar la confiabilidad de la prueba. ${ }^{22,23}$

De acuerdo con los resultados obtenidos en nuestro estudio, las mejores pruebas para el diagnóstico temprano de IVU son la determinación de la bacteriuria en orina centrifugada, esterasa leucocitaria y la cuenta de leucocitos, debido a su alta especificidad y sensibilidad. Esto coincide con lo notificado en un metaanálisis ${ }^{24}$ realizado con el propósito de determinar la confiabilidad del uroanálisis para el diagnóstico de IVU; en éste se encontró una alta sensibilidad (99.8\%) en la combinación de esterasa de leucocitos, nitritos y microscopía positiva, en muestras de orina no centrifugada, y la mayor especificidad la demostró la determinación de nitritos (98\%). En el presente estudio, la prueba de nitritos obtuvo una especificidad de $100 \%$ y una sensibilidad de $36 \%$; resultó positiva en todos los casos en los que se detectaron bacterias reductoras de nitratos por urocultivo, y fue específica para este tipo de microorganismos.

Los resultados que se presentan, muestran que la confiabilidad de tres o más pruebas del uroanálisis incrementa la sensibilidad $(84.3 \%)$, la especificidad (100\%), el VPP (100\%) y el VPN (93.2\%). Al comparar estos valores con lo informado por Lumbiganon y colaboradores para la tira reactiva global, se observan diferencias en la sensibilidad y en el VPP que pueden atribuirse a la mayor sensibilidad de los métodos que se emplean en la determinación por microscopía de bacteriuria y cuenta de leucocitos.
Los resultados del presente estudio indican que la determinación de bacteriuria en sedimento urinario teñido con el método de Gram presenta una sensibilidad y a una especificidad elevadas (100 y 81.6\%, respectivamente); por ese motivo, se recomienda la realización y la evaluación de esta prueba en la práctica rutinaria del análisis de orina, ya que habitualmente no se realiza en los laboratorios de diagnóstico clínico $y$, junto con la determinación de nitritos y esterasa leucocitaria, puede ser un buen indicador en el diagnóstico temprano de las IVU y contribuir a orientar y mejorar el tratamiento inicial con los resultados del urocultivo y la prueba de sensibilidad a los antibióticos. La detección de bacterias en frotis de sedimento urinario permite la diferenciación entre bacterias Gram positivas y Gram negativas, así como entre cocos y bacilos, lo cual puede orientar la toma de decisiones respecto al tratamiento. Es factible realizar esta prueba rápida, sencilla y de bajo costo en la práctica diaria del laboratorio clínico.

Una limitante de este estudio fue el tamaño de la muestra, lo cual impide extrapolar los resultados al total de la población diabética; no obstante, éstos pueden ser base de otras investigaciones y un indicador que oriente hacia el uso rutinario de pruebas valiosas por su sencillez y la prontitud con la que aportan información.

El uroanálisis no puede sustituir al urocultivo en la confirmación del diagnóstico y la identificación del agente etiológico de las IVU, pero las pruebas presuntivas pueden apoyar una mejor toma de decisiones al tratar pacientes que, por su estado, no pueden esperar a iniciar el tratamiento hasta los resultados del urocultivo (48, como mínimo). 


\section{Referencias}

I. Naber KG, Bergman B, Bishop MC, Bjerklund-Johanuen TE, Botto H, Lobel $B$ et al. EAU guidelines for the management of urinary and male genital tract infections. Urinary Tract Infection (UTI) Working Group of the Health Care Office ( $\mathrm{HCO}$ ) of the European Association of Urology (EAU). Eur Urol 200I;40(5):576-588.

2. Nicolle LE. A practical guide to antimicrobial management of complicates urinary tract infection. Drugs Aging 2001;18(4):243-254. 3. Wullt B, Bergsten G, Fisher H.Application of laboratory research in UTI. EAU Update Series 2(2004): I 16-I 24.

4. Cabezas-Agrícola JM,Touriño Perlaba R, Cabezas-Cerrato J.

Complicaciones crónicas de la diabetes mellitus. Medicine 2004:9(16):990-999.

5. Boyko EJ, Fihn SD, Scholes D, Chen CL, Normand EH, Yarbro P. Diabetes and the risk of acute urinary tract infection among postmenopausal women. Diabetes Care 2002;25:1778- 1783. 6. Ronald A, Ludwig E. Urinary tract infections in adults with diabetes. Int J Antimicrob Agents 2001;17(4):287-292.

7. Stapleton A. Urinary tract infections in patients with diabetes. Am J Med 2002; I I 3 Suppl IA:80S-84S.

8. Hernández-Mijares A, Morillas-Ariño C, Lluch-Verdú I, Riera-Fortuny C, Graña-Fandos J, Iñigo-Zaera P et al. Infección y diabetes. Semergen 2000;24 (7):539-544

9. Olaiz G, Rojas R, Barquera S, Shamah T, Aguilar C, Cravioto P et al. Encuesta Nacional de Salud 2000. La salud de los adultos. Cuernavaca, Morelos, México; Instituto Nacional de Salud Pública, 2003;52:94- 103. 10. Secretaría de Salud. Principales causas de mortalidad general 2003. México DF, SSA, 2005. Disponible en: http://www.salud.gob.mx. II. Goswami R, Bal CS, Tejaswi S, Punjabi GV, Kapil A, Kochupillai N. Prevalence of urinary tract infection and renal scars in patients with diabetes mellitus. Diabetes Res Clin Pract 200 I;53(3):I8I-I86.

12. Geerlings SE, Stolk RP, Camps MJ, Netten PM, Hoekstra JB, Bouter $\mathrm{KP}$ et al. Asymptomatic bacteriuria may be considered a complication in women with diabetes. Diabetes Mellitus Women Asymptomatic Bacteriuria Utrecht Study Group. Diabetes Care 2000;23(6):744-749.
13. Kehinde EO, Rotimi VO,Al-Awadi KA, Abdul-Halim H, Boland F,AlHunayan $A$ et al. Factors predisposing to urinary tract infection after ureteral stent insertion. J Urol 2002;167(3):I334-I337.

14. Aguirre-Avalos G, Zavala-Silva ML, Diaz-Nava A,Amaya-Tapia G, Aguilar-Benavides S. Asymtomatic bacteriuria and inflammatory response to urinary tract infection of elderly ambulatory women in nursing homes. Arch Med Res 1999;30(1):29-32.

15. Laso MC. Interpretación del análisis de orina. Arch Argent Pediatr 2002; 100(2): I79-183.

16. Lumbiganon P, Chongsomchai C, Chumworathayee B, Thinkhamrop J. Reagent strip testing is not sensitive for the screening of asyntomatic bacteriuria in pregnant women.J Med Assoc Thai 2002;85(8):922-927. 17. Tincello DG, Richmond DH. Evaluation of reagent strips in detecting asymptomatic bacteriuria in early pregnancy: Prospective case series. BMJ 1998;316(7I29):435-437.

18. Uncu Y, Uncu G, Esmer A, Bilgel N. Should asymptomatic bacteriuria be screened in pregnancy? Clin Exp Obstet Gynecol 2002;29(4):281285.

19. Nunns D, Smith AR, Hosker G. Reagent strip testing urine for significant bacteriuria in a udorynamic clinic. Br J Urol 1995;76(I):87-89. 20. Etherington IJ, James DK. Reagent strip testing of antenatal urine specimens for infection. Br J Obstet Gynaecol 1993;100(9):806-808. 2I. Leanos-Miranda A, Contreras-Hernández I, Camacho R, VillagómezSalcedo $E$, Cervantes-Gorayeb I. Diagnóstico de infecciones del tracto urinario por varias pruebas urinarias. Rev Invest Clin 1996;48(2): I I7123.

22. Shaw KN, McGowan KL, Gorelick MH, Schwartz JS. Screening for urinary tract infection in infants in the emergency department: Which test is best? Pediatrics 1998;101(6):150-157.

23. Brauner A, Flodin U, Hylander B, Ostenson CG. Bacteriuria, bacterial virulence and host factors in diabetic patients. Diabet Med 1993;10(6):550-554.

24. American Academy of Pediatrics. Committee on Quality Improvement. Practice parameter:The diagnosis, treatment, and evaluation of the initial urinary tract infection in febrile infants and young children. Pediatrics 1999;103:843-852. 